Supporting Information for

\title{
L-cysteine-Assisted Synthesis of PbS Nanocube-Based Pagoda-like Hierarchical Architectures
}

\author{
Fan Zuo, Si Yan, Bin Zhang, Yu Zhao and Yi Xie* \\ Department of Nanomaterials and Nanochemistry, Hefei National Laboratory for Physical Sciences \\ at Microscale, University of Science and Technology of China, Hefei, Anhui 230026 (P. R. China) \\ Email: yxie@ustc.edu.cn
}

Figure S1. EDAX analysis of the $\mathrm{PbS}$ pagoda-like hierarchical architectures. The atom ratio of $\mathrm{Pb}: \mathrm{S}$ element is c.a. 1: 1, consistent with stoichiometric proportion of $\mathrm{PbS}$.

Figure S2. XRD pattern of the precursor collected from the mixed L-cysteine and $\mathrm{Pb}(\mathrm{Ac})_{2}$ suspension. Contrasted with the XRD pattern of $\mathrm{PbS}$ (Figure 1), No characteristic diffraction peaks of $\mathrm{PbS}$ is found. 